\title{
Fe-25Mn-3Al-3Si TWIP-TRIP Steel Deformed at High Strain-Rates
}

\author{
J. T. Benzing ${ }^{1}$, J. E. Wittig ${ }^{1}$, T. M. Smith², M. J. Mills², J. R. Johnson², G. S. Daehn², J. Bentley ${ }^{3}$, \\ D. Raabe ${ }^{4}$, C. Ophus ${ }^{5}$ \\ ${ }^{1 .}$ Interdisciplinary Materials Science, Vanderbilt University, Nashville TN 37232, USA \\ 2. Materials Science and Engineering, The Ohio State University, Columbus, OH 43210, USA \\ 3. Microscopy and Microanalytical Sciences, PO Box 7103, Oak Ridge, TN 37831-7103, USA \\ 4. Max-Planck-Institut für Eisenforschung, Max-Planck-Straße 1, D-40237 Düsseldorf, Germany \\ 5. National Center for Electron Microscopy, Molecular Foundry, Lawrence Berkeley National Lab, \\ Berkeley, CA 94720, USA
}

High-Mn steels exhibit high strength, superior strain hardening and high formability, which are attractive to the automotive industry for reduced weight, crashworthiness and cold stamping of complex parts [1]. When $\mathrm{Fe}$ is alloyed with $25 \% \mathrm{Mn}, 3 \% \mathrm{Al}$ and $3 \% \mathrm{Si}(\mathrm{wt} \%)$, these substitutional elements stabilize the gamma phase, enhance the solid-solution strengthening effect, and reduce the stacking fault energy (SFE). Strain hardening in this alloy involves the development of secondary deformation mechanisms such as twinning-induced plasticity (TWIP) and transformation-induced plasticity (TRIP), that allow for uniform elongation up to $80 \%$ in a room-temperature (RT) tensile test at quasi-static strain rates $\left(\sim 10^{-4} \mathrm{~s}^{-1}\right)$. A SFE of $21 \pm 3 \mathrm{mJm}^{-2}$, which was experimentally determined [2] for this alloy using weak-beam dark-field transmission electron microscopy (TEM) and incorporated elastic constants measured by a novel combination of orientation imaging microscopy and nano-indentation [3], promotes TWIP as the dominant mode of RT secondary deformation with some evidence of TRIP in the form of hexagonal $\varepsilon$-martensite. In the current work, uniaxial ring expansion that utilized a shock wave from a vaporized wire provides strain rates from $\sim 10^{3}$ to $10^{4} \mathrm{~s}^{-1}$. Since adiabatic heating is a product of higher strain rates and SFE is sensitive to temperature, understanding the deformation mechanisms as a function of strain rate requires quantitative characterization of the microstructural evolution [4].

The strain rate from the ring expansion can be controlled by ring diameter and strain by input energy. With radius-to-thickness ratios held constant, inner-ring diameters of $51,25, \& 16 \mathrm{~mm}$ resulted in strain rates of $1100,3000 \& 9000 \mathrm{~s}^{-1}$ and input energies of $8,4 \& 2 \mathrm{~kJ}$ produced total strains $\varepsilon_{\text {true }}=20,14 \&$ $12 \%$, respectively, for each size. TEM samples were sectioned orthogonal to the ring outer radius with electro-discharge machining to produce $3 \mathrm{~mm}$ diameter discs, which were electropolished to electron transparency with a Struers twin-jet system $\left(20 \% \mathrm{HNO}_{3} / 80 \% \mathrm{CH}_{3} \mathrm{OH},-30{ }^{\circ} \mathrm{C}, 15 \mathrm{~V}\right)$. A Philips CM20 was used to survey the samples in order to locate grains with secondary deformation habit planes parallel to the $\langle 110\rangle$ beam direction. As was observed for RT deformation at a quasi-static strain rate, many grains were characterized by multiple twin/martensite variants as well as termination of stacking faults at twin/martensite interfaces. Grains selected for high-resolution (HR) imaging met the criteria of minimal alpha/beta tilt, so as to minimize effective sample thickness. Figure 1 shows results from the 50 $\mathrm{mm}$ dia. ring expanded to $\varepsilon_{\text {true }}=20 \%$ at $\dot{\varepsilon}=1100 \mathrm{~s}^{-1}$, where selected area electron diffraction (SAED) patterns indicate the presence of either TRIP or TWIP in separate grains of the same TEM disc. These two grains were imaged in high-angle annular dark-field (HAADF) STEM mode with a probe-corrected FEI Titan and in HRTEM mode with an image-corrected FEI Titan. The HAADF-STEM images provided the most useful data as the HRTEM phase-contrast was complicated by variations in sample thickness, crystal orientation due to bending of the foil and objective-lens defocus. Although the SAED patterns in Figure 1 indicated either TRIP or TWIP, the HAADF images in Figure 2a-b show that both twinning and hexagonal stacking are present together in the same grain with widths on the order of a few nanometers, regardless of mechanism that dominated the SAED pattern. Preliminary results from strain mapping of the HR-STEM images using a real-space lattice method that combines images taken at 0 and $90^{\circ}$, with respect to the slow-scan direction, reveal significant shear strains at twin and martensite interfaces. Quantitative strain measurements at twin and martensite interfaces may provide insight into the role of secondary deformation mechanisms on the strain hardening behavior of these high-Mn austenitic steels [5]. 
[1] O Bouaziz et al," Curr. Opin. Solid State Mater. Sci., (2011).

[2] D T Pierce, J A Jiménez, J Bentley, D Raabe, C Oskay and J.E. Wittig, Acta Mater 68(2014)238-53

[3] D T Pierce, K Nowag, A Montagne, J A Jiménez, J E Wittig and R Ghisleni, Mater Sci Eng A578(2013)134-9

[4] Pierce, Dean Thomas, Ph.D dissertation, Vanderbilt University, (2014).

[5] This work was funded by the US National Science Foundation Division of Materials Research under grant DMR1309258, the MPIE in Düsseldorf and the OSU in Columbus (MSE dept. and CEMAS).

Work at the Molecular Foundry was supported by the Office of Science, Office of Basic Energy

Sciences, of the U.S. Department of Energy under Contract No. DE-AC02-05CH11231.
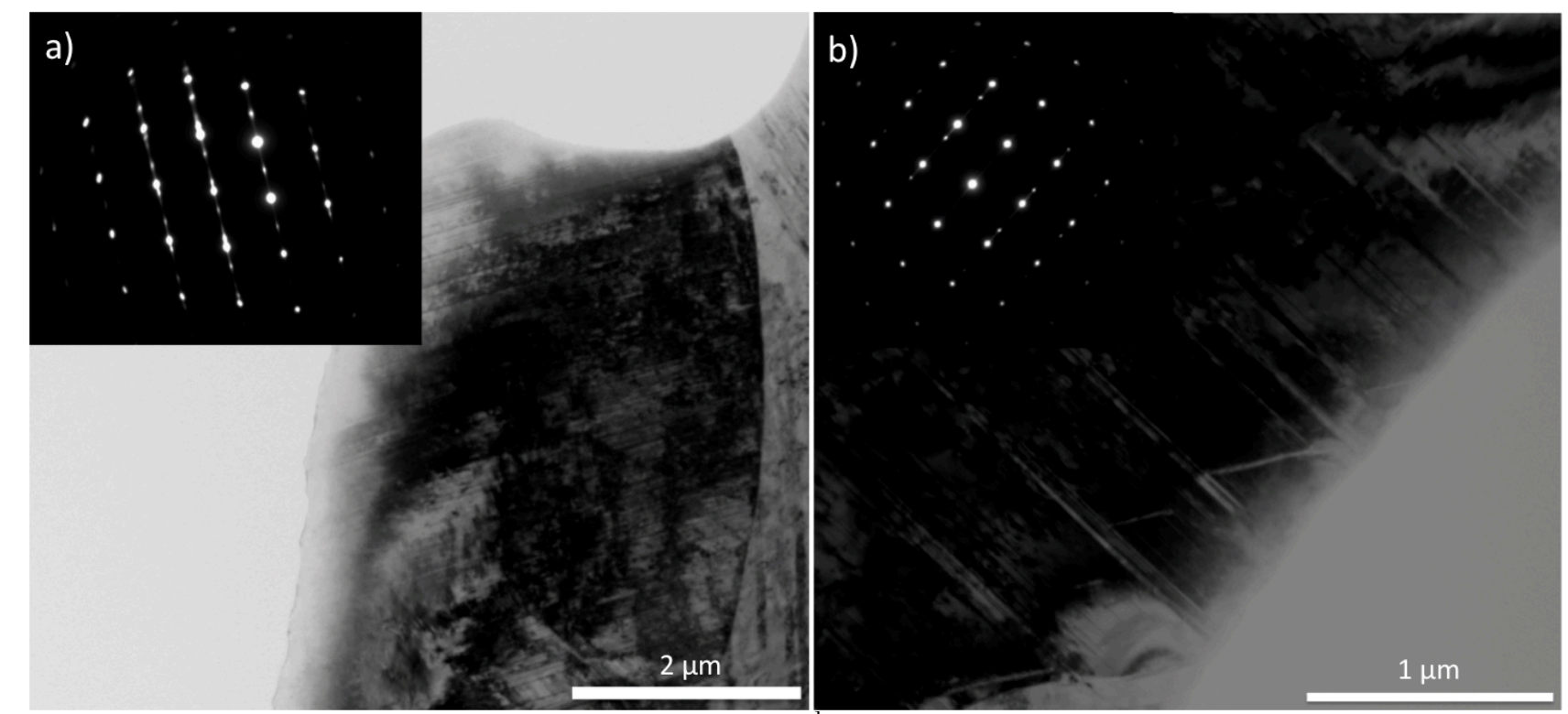

Figure 1: BF TEM images of $\varepsilon_{\text {true }}=20 \%, \dot{\varepsilon}=1100 \mathrm{~s}^{-1}$. The inset SAED patterns, recorded at $<110>$ zones, indicate two modes of secondary deformation: a) TRIP b) TWIP.

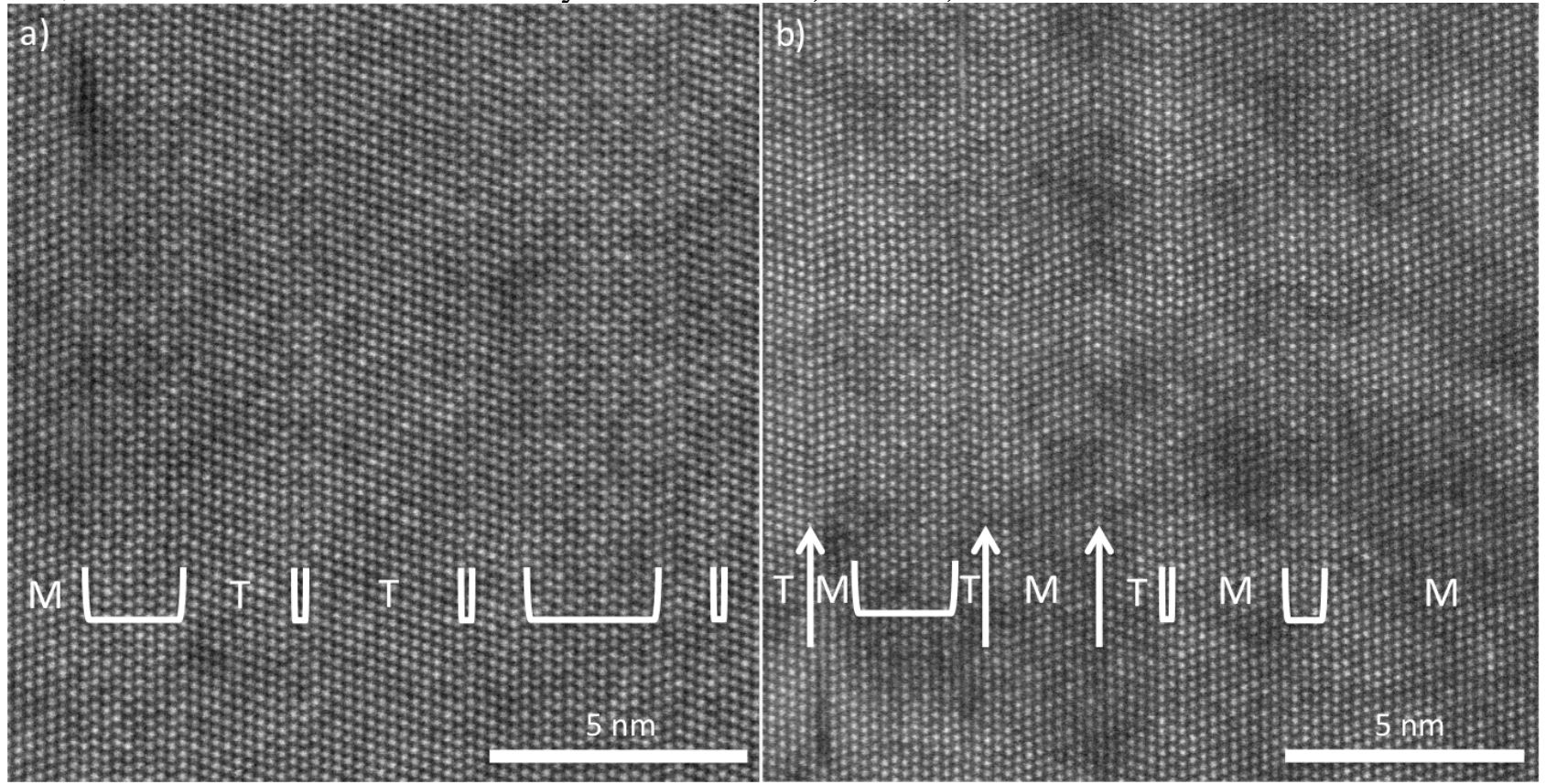

Figure 2: High-resolution HAADF-STEM images recorded using a probe-corrected FEI Titan from the respective grains in Figure 1, where regions of matrix $(\mathrm{M}), \varepsilon$-martensite platelets (bracket), twinning with respect to matrix (T) and twin boundaries (arrow) are marked: a) SAED with martensite reflections (TRIP) b) SAED with twin reflections (TWIP). 